in Malta, were analysed for sources of error. The British National Formulary for Children (BNFC) was used to establish the correct prescribing standard.

Methods Treatment charts of all admissions to medical paediatric inpatient wards were reviewed daily over a fourweek period. Prescriptions for Paracetamol were assessed for legibility, inedibility, approved drug nomenclature, correct dose and dosing frequency, approved dosing interval abbreviations, writing of minimum dosing interval for pro re nata (PRN), appropriate dating, prescriber signature and prescriber designation. Treatment charts were also analysed to assess accurate writing of patient name, identification number, age, date of birth, height, weight, and allergies. Paracetamol prescriptions for indications other than fever were excluded.

Results A total of 72 treatment charts were analysed of which 44 contained Paracetamol prescriptions. Age ranged from 1 day to 13 years. $93.2 \%$ of all prescriptions were on a PRN basis. Legibility and inedibility met the BNFC standard in $100 \%$ of cases. Approved drug nomenclature was used in 97.7\% of prescriptions.

With regards to dosing, $54.5 \%$ of prescriptions did not follow the standard leading to incorrect dosing. Of these cases, $50 \%$ were due to the same dose of Paracetamol being prescribed for the oral, intravenous and rectal routes used for the same child. In the other 50\%, the oral dose was calculated by weight instead of using fixed dose ranges based on age. Where errors were made, patients were overdosed by an average of $20 \%$ more than the recommended maximum dose. Correct dosing frequency was present in $100 \%$ of cases. $97.7 \%$ of dosing interval abbreviations were not according to guidelines, mainly because English abbreviations were not written in full. PRN was not written in $51.2 \%$ of Paracetamol PRN prescriptions.

Dating was correct in $95.5 \%$ of cases. Prescriber signature present in $97.7 \%$ of cases and prescriber designation in 95.5\% of prescriptions.

The following table illustrates data related to treatment charts:

\begin{tabular}{ll} 
Abstract 225 Table 1 & \\
\hline Patient Criteria & $\begin{array}{l}\text { Percentage Correctly } \\
\text { Completed }\end{array}$ \\
\hline Name & 94.4 \\
Identification Number & 94.4 \\
Age & 83.3 \\
Birth date & 22.2 \\
Height & 1.4 \\
Weight & 76.4 \\
Allergies & 54.2 \\
\hline
\end{tabular}

Conclusions Adherence to proper prescription practices has been repeatedly emphasised by regulatory bodies and safe prescription is considered an integral part of sound medical practice. Greater attention needs to be paid to appropriate dosing according to indication and route to prevent overdosing. Correct writing of dosing interval abbreviations, as well as recording of weight and allergies in treatment charts is also essential.

\section{MIND THE GAP!! UNCOMMON CAUSE OF HIGH ANION GAP METABOLIC ACIDOSIS DUE TO PYROGLUTAMIC ACIDOSIS (PGA)}

Alagusutha Jeyaraman, Neil Wright, Marta Cohen. UK

\subsection{6/bmjpo-2021-RCPCH.124}

Background Metabolic acidosis is common in sick children. Measurement of anion gap helps to narrow down the cause of high anion gap metabolic acidosis such as DKA/lactic acidosis/renal failure. A rare cause of high anion gap metabolic acidosis is due to accumulation of pyroglutamic acid. Acquired PGA occurs in association with glutathione and cysteine depletion or as a side effect of certain medications. We report a complex case of child with GLIS3 mutation who had high anion gap metabolic acidosis secondary to acquired pyroglutamic aciduria associated with common drugs such as paracetamol.

\section{Objectives}

Methods

Results 10 yrs old girl was born at 35 weeks gestation with severe growth retardation with birth weight of $1.17 \mathrm{~kg}$. She developed neonatal diabetes on day 3 and congenital hypothyroidism on day 4. The combination of neonatal diabetes, congenital hypothyroidism and some dysmorphic facial features led to a clinical diagnosis of a GLIS 3 mutation, subsequently confirmed by genetic analysis.Her neonatal diabetes was managed with insulin pump therapy and high dose thyroxine in order to maintain her thyroid function. She was treated for exocrine pancreatic insufficiency, gastro oesophageal reflux with jejunostomy feeds. She also had liver transplantation at the age of 3 yrs for hepatic fibrosis and cirrhosis. She had polycystic kidney disease and deteriorating renal function, hypertension, chronic anaemia, recurrent chest infections and developmental delay. She was admitted with a viral illness and chest infection treated with cefuroxime, paracetamol and salbutamol along with her regular diabetes and thyroid medicines. She was initially managed in HDU, needed high flow oxygen support and strict fluid balance. In the ward, she suddenly became unresponsive and collapsed with an asystolic cardiac arrest. She developed severe metabolic acidosis with very high anion gap. The anion gap suggested the presence of a fixed acid and was not due to ketone production related to diabetes. This was confirmed at post-mortem also. She received prolonged resuscitation with multiple doses of adrenaline and boluses of bicarbonate. But remained in asystole and sadly succumbed.The pathologist noted that the child had a high anion gap metabolic acidosis and subsequent post mortem organic acid profile suggested that it was due to pyroglutamic aciduria. Pyroglutamic acidosis is noted to be a rare phenomenon which can happen in acutely unwell patients who receive antibiotics and paracetamol. The child had received paracetamol at a reduced dose in view of her renal impairment.

Conclusions The learning point from the above presentation is that Pyroglutamic acidosis (PGA) should be considered as a differential diagnosis in patients presenting with high anion gap metabolic acidosis with complex co-morbidities and risk factors. The mainstay of treatment of PGA includes cessation of medications that can influence the $\gamma$-glutamyl cycle or glutathione levels, N-Acetyl Cysteine (NAC) and the implementation of supportive measures. Unrecognised acidosis due to PGA can contribute to morbidity and mortality in patients with hepatic and renal dysfunction. 